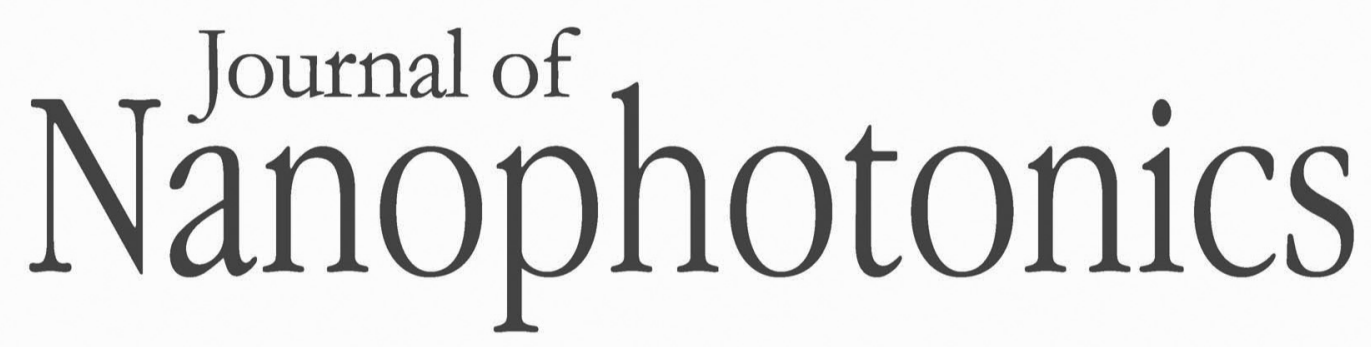

Ultra-short-pulsed laser-machined nanogratings of laser-induced periodic surface structures on thin molybdenum layers

Davide Scorticati

Gert-Willem Römer

Dirk Frederik de Lange

Bert Huis in 't Veld 


\title{
Ultra-short-pulsed laser-machined nanogratings of laser-induced periodic surface structures on thin molybdenum layers
}

\author{
Davide Scorticati, ${ }^{a}$ Gert-Willem Römer, ${ }^{a}$ Dirk Frederik de Lange, ${ }^{c}$ and \\ Bert Huis in ' $t$ Veld ${ }^{\mathrm{a}, \mathrm{b}}$ \\ ${ }^{a}$ University of Twente, Faculty of Engineering Technology, 7500AE Enschede, Netherlands \\ D.Scorticati@utwente.nl

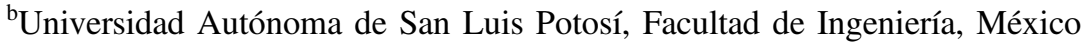 \\ ${ }^{\mathrm{c}}$ TNO Technical Sciences, Mechatronics, Mechanics and Materials, 5600AE Eindhoven,
} Netherlands

\begin{abstract}
Large areas of regular diffraction nanogratings were produced consisting of so-called laser-induced periodic surface structures (LIPSS) on thin molybdenum layers $(<400 \mathrm{~nm})$ deposited on a borosilicate glass substrate. The aim was to produce these structures without ablating nor cracking the molybdenum layer. Ultra short laser pulses were applied using a focused Gaussian beam profile. Processing parameters such as laser fluence, pulse overlap, number of overscans, repetition frequency, wavelength and polarization were varied to study the effect on periodicity, height, and especially regularity of the obtained LIPSS. It was found that a careful choice of the correct laser parameters is required to avoid detrimental mechanical stresses, cracking, and delamination during the laser processing of the layer in order to remain in its correct range of ductility as well as to ensure regular LIPSS. A possible photovoltaic application of these nanogratings could be found in texturing of thin film cells to enhance light trapping mechanisms. (c) 2012 Society of Photo-Optical Instrumentation Engineers (SPIE). [DOI: 10.1117/1.JNP.6.063528]
\end{abstract}

Keywords: ultra short laser pulses; surface processing; brittle to ductile transition; molybdenum; laser-induced periodic surface structures; $p s$ laser; nanogratings; copper indium gallium selenide solar cells; textured back-contact.

Paper 12072P received Jun. 20, 2012; revised manuscript received Nov. 6, 2012; accepted for publication Nov. 6, 2012; published online Dec. 3, 2012.

\section{Introduction}

Laser-induced periodic surface structures (LIPSS), also referred to as ripples, are wavy structures observed on the surface of many materials after laser irradiation. Although LIPSS have been studied since $1965,{ }^{1}$ their exact origin is still debated. However, it is well-known that the regularity, shape and dimensions of LIPSS depend on the laser irradiation conditions as well as material properties. Periodicity, amplitude and orientation of LIPSS depend on the wavelength of the laser radiation, fluence, polarization, angle of incidence and the refractive index of the material. ${ }^{2-4}$ Under most laser conditions, LIPSS show bifurcations and/or forking-i.e. when a single ripple splits into two ripples of half periodicity — and their length is limited to the diameter of the laser spot. Interestingly, by choosing proper laser parameters, the length of LIPSS can be extended without bifurcations or forking to areas larger than the spot size, as has been shown on bulk materials. ${ }^{5}$ A practical application of LIPSS can be found in diffraction nanogratings. ${ }^{6}$

In the present work, we investigated the feasibility of nanogratings consisting of LIPSS on thin molybdenum (Mo) layers of a thickness $\leq 400 \mathrm{~nm}$. The challenge, while texturing a thin molybdenum film, is to find the proper scanning conditions for obtaining a uniform area of LIPSS - which occur only in a limited range of total deposited fluence on a given material (see Ref. 7)—and for avoiding thermo-mechanical damage during the laser treatment. The latter includes cracking, delamination, and excessive ablation of the thin layer.

0091-3286/2012/\$25.00 @ 2012 SPIE 


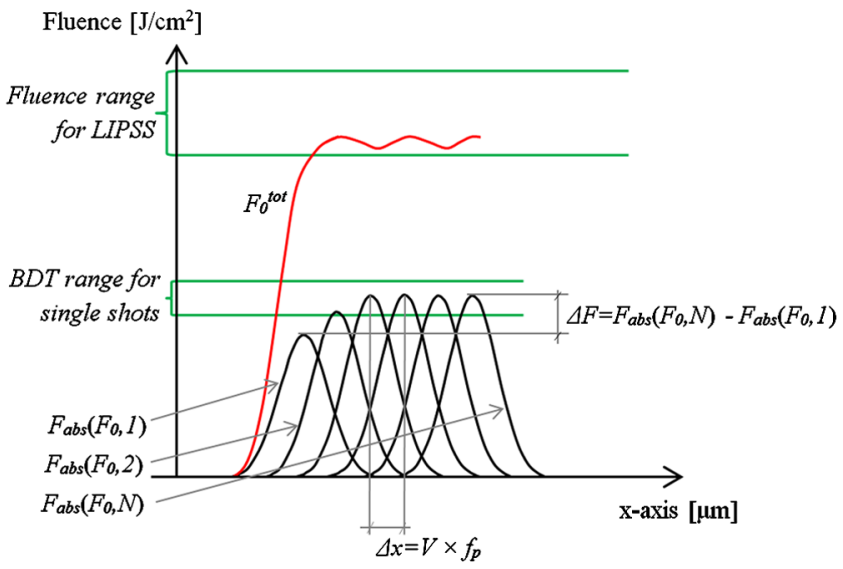

Fig. 1 For creating LIPSS on thin Mo layers without thermal damage, two requirements need to be fulfilled: (i) The absorbed fluence Fabs(FO,N) of each subsequent laser pulse shall be in the BDT range not to thermally damage the layer, and (ii) the accumulated fluence Fotot being in the range for obtaining LIPSS. ${ }^{7}$

To avoid thermo-mechanical damage on a thin molybdenum film, the so-called brittle to ductile transition (BDT) plays a primary role and a careful choice of the correct single pulse fluence is required well below the total-or accumulated - fluence range needed for creating LIPSS, as will be shown in Fig. 1. The particular behavior of the BDT on thin metallic layers during laser irradiation was first discussed for laser removal of thin chromium films by Lee et al. and Siegel et al. using an excimer laser. ${ }^{8,9}$

When compared to other metals, in body centered cubic (bcc) lattice metals such as molybdenum, chromium and tungsten, the ductile range is reached abruptly above a certain temperature..$^{9-11}$ In such materials, because of the BDT, excessive thermal stresses can be avoided during the cooling down phase only when high temperature gradients lay in the ductile region above the BDT. For this reason, the thin molybdenum layer only can withstand thermal stresses during the laser treatment without cracking in the case where the absorbed fluence level is above a certain threshold. Khantha et al. ${ }^{11}$ discussed, theoretically, the connection between the high generation of laser induced defects below the melting temperature and the BDT threshold temperature. However, if the absorbed fluence is too high, excessive ablation or chipping/delamination occurs. Therefore, damage-free surface texturing is possible only within a certain absorbed fluence range.

Moreover, when applying high spatial pulse-to-pulse overlap, the absorptivity of the material to laser energy varies as a function of the number of pulses and the laser fluence. After the first shots, incubation effects, due to laser-induced surface defects and increased roughness, ${ }^{12}$ lead to a lower surface reflectivity. The latter leads to an increased absorption of laser radiation and to subsequent thermal damage [as will be shown in Sec. 3.1.1, Fig. 2(c) and 2(d)]. The absorbed fluence $F_{\text {abs }}\left(\mathrm{J} / \mathrm{cm}^{2}\right)$ can be expressed as

$$
F_{\text {abs }}\left(F_{0}, N\right)=F_{0} \times A\left(F_{0}, N\right),
$$

where $F_{0}\left(\mathrm{~J} / \mathrm{cm}^{2}\right)$ is the incident laser fluence and $A\left(F_{0}, N\right)$ is the changing absorptivity as function of the number of applied laser pulses $N$ and the impinging averaged laser fluence $F_{0}$.

\section{Experimental Setup}

\subsection{Laser Setup}

Two different laser sources were employed to study the effect of fluence, pulse duration and wavelength on LIPSS formation in the molybdenum layer:

- the Pharos from Light Conversion Ltd., which is a Yb:KGW laser source, central wavelength of $1030 \mathrm{~nm}$ infrared (IR), with tunable pulse duration (ranging from 230 fs to $10 \mathrm{ps}$ ), 

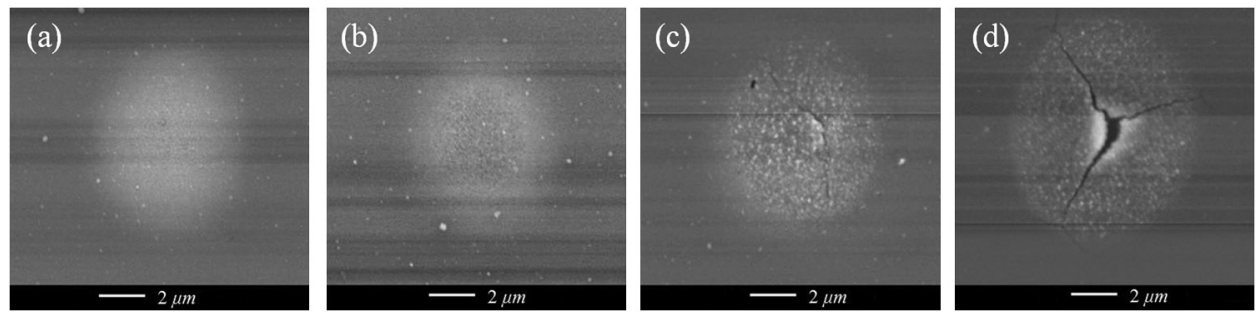

Fig. 2 SEM pictures of the surface after multiple laser shots at a fixed fluence of $0.07 \mathrm{~J} / \mathrm{cm}^{2}$, with fixed pulse duration of $6.7 \mathrm{ps}$, obtained at UV wavelength, fixed spot diameter of $12 \mu \mathrm{m}$ and at different overscans on $150 \mathrm{~nm}$ molybdenum layer: (a) 1 shot, (b) 2 shots, (c) 5 shots and (d) 10 shots. This sequence shows that the fluence range to process the layer free of any damage, is changed due to the enhanced absorption, as described by Eq. (1). The latter is the result of increased roughness and subsurface defects induced by subsequent laser shots. The evidence of this statement is given by the different color of the laser-treated surface, due to different material properties and is well explained by Jee et al. ${ }^{12}$

maximum repetition frequency $200 \mathrm{kHz}, 10 \mathrm{~W}$ maximum average output power (in IR) at $200 \mathrm{kHz}$ and a beam quality of $M^{2}<1.2$.

- the TruMicro 5050 from Trumpf GmbH, which is a Yb:YAG laser source, central wavelength of $1030 \mathrm{~nm}$ (IR), with fixed pulse duration of $6.7 \mathrm{ps}$, maximum repetition frequency of $400 \mathrm{kHz}, 50 \mathrm{~W}$ maximum average output power (in IR) at $400 \mathrm{kHz}$ and $M^{2}<1.3$.

Both laser sources showed a Gaussian power density distribution and were equipped with a pulse-picker, allowing the adaptation of the repetition frequency without affecting the energy per pulse. To study the effect of the wavelength, a second harmonic generation or a third harmonic generation unit was employed to convert the central wavelength to $515 \mathrm{~nm}$ (green) or to $343 \mathrm{~nm}$ ultraviolet (UV).

In both systems, galvano-scanners (IntelliScan14 of Scanlab GbmH) were used to manipulate the focal spot over the surface of the sample. The scanners were equipped with telecentric $F$-theta lenses (Ronar of Linos $\mathrm{GmbH}$ ) to focus the laser beam on the samples. In the case of the TruMicro laser source, the focal length of theta lenses used for IR, green, and UV were, respectively 80,100 , and $100 \mathrm{~mm}$, while in the case of the Pharos source, only the IR wavelength was exploited and at a focal length of the lens of $100 \mathrm{~mm}$. All experiments were carried out at normal incidence of the laser beam on the surface and at environmental conditions.

The optical $1 / e^{2}$ diameter $D(m)$ of the focused laser beam on the surface was determined using the " $D^{2}$ method" for each wavelength used. ${ }^{4}$ This diameter was found to range from 12 to $30 \mu \mathrm{m}$, depending on the wavelength and setup used. Given the spot diameter $D$, the average laser fluence $F_{0}$-here defined as: $F_{0}=E_{0} /\left(\pi D^{2}\right)$, where $E_{0}$ is the pulse energy-was then calculated for single pulses. The effect of spatial pulse-to-pulse laser spot overlap (OL) on LIPSS formation was also studied. We define the term OL as the physical displacement distance of the laser spot between successive pulses: ${ }^{13}$

$$
\mathrm{OL}=1-v /\left(D \times f_{p}\right)
$$

where $v(\mathrm{~m} / \mathrm{s})$ denotes the velocity of the laser spot relative to the substrate, and $f_{p}(\mathrm{~Hz})$ the repetition frequency of the laser source. Note that, for increasing velocity, OL goes to zero, or even will becomes negative, indicating no spatial overlap between subsequent laser pulses on the surface.

\subsection{Analysis Equipment}

Morphological inspection of the laser-treated areas was performed by optical microscopy, scanning electron microscopy (SEM, JCM-5000 NeoScope), atomic force microscopy (AFM, Nanosurf Easyscan 2) and confocal laser scanning microscopy (CLSM, Keyence VK-9700). A spectrophotometer (PerkinElmer Lambda 950 with ARTA accessory) was used to analyze the angular intensity distribution (AID) of the refracted light at different angles and wavelengths of the obtained nanogratings. ${ }^{14}$ 


\subsection{Samples}

Molybdenum layers of different thickness were deposited on glass substrates. For initial tests, samples of $150 \mathrm{~nm}$ thickness deposited by physical vapor deposition (PVD) on borosilicate glass were used. For structuring larger areas with LIPSS, molybdenum layers deposited by sputtering on soda lime glass by a third party were used. The latter combination of thin film molybdenum on soda lime glass is meant for the production of $\mathrm{Cu}(\mathrm{In}, \mathrm{Ga}) \mathrm{Se}_{2}$ solar cells. The molybdenum for this application has, in general, a high porosity, ${ }^{15}$ while molybdenum for other applications might require more dense layers and will react differently during laser texturing. The latter is not addressed in this work.

\section{Experimental Results and Discussion}

\subsection{Experimental Procedure}

The experimental procedure to determine the processing window for damage-free LIPSS on the thin molybdenum layers can be divided into three steps:

1. First, determining processing conditions for single laser pulses (Sec. 3.1.1). This section will show the importance of reaching the BDT transition for each individual pulse, while texturing thin molybdenum layers as a function of the laser fluence.

2. Next, determining processing conditions for (partly) spatial overlapping pulses (Sec. 3.1.2) resulting in lines, or tracks with LIPSS. This section will show the effect of processing parameters-fluence, pulse overlap, pulse frequency, pulse duration, number of overscans and wavelength - on the characteristics of LIPSS. As well as the importance of ensuring that the total (accumulated) fluence is in the right range for creating LIPSS.

3. Finally, determining the optimal pitch between laser tracks, and polarization direction required to generate uniform areas of bifurcation-free and forking-free LIPSS.

\subsubsection{Single laser shots}

Figure 3 shows the surface morphology of a $150 \mathrm{~nm}$-thick molybdenum layer on glass after laser processing, as function of laser fluence ranging from 0.05 to $0.09 \mathrm{~J} / \mathrm{cm}^{2}$. If the applied laser fluence is too low, the layer is not able to withstand thermally induced stresses and will crack, as seen in Fig. 3(a). That is, for a fixed molybdenum film thickness, ${ }^{8}$ below a certain fluence threshold the material will not reach the BDT, and the layer will crack. Above this threshold the BDT is reached and the ductility of the layer allows it to cope with thermal stresses, as seen in Fig. 3(b). If the laser fluence is too high, the layer will be chipped off, as in Fig. 3(c). It should be noted that the fluence range for damage-free processing is small. These results are comparable to those shown by Lee et al. ${ }^{8}$ and Siegel et al. ${ }^{9}$ on thin chromium layers.
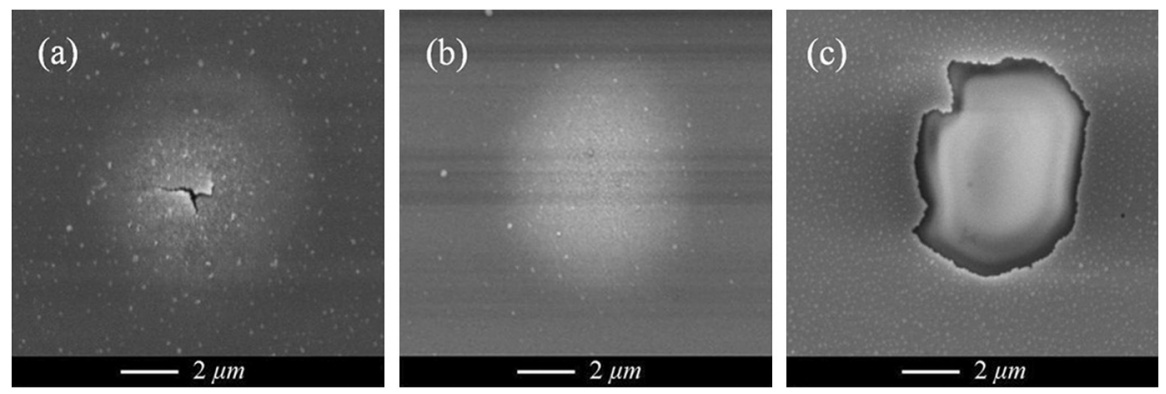

Fig. 3 SEM pictures of the surface after single laser shots at different fluence levels, with fixed pulse duration of $6.7 \mathrm{ps}$, obtained using UV wavelength and fixed spot diameter of $12 \mu \mathrm{m}$ on $150 \mathrm{~nm}$ molybdenum layer: (a) $0.05 \mathrm{~J} / \mathrm{cm}^{2}$, (b) $0.07 \mathrm{~J} / \mathrm{cm}^{2}$ and (c) $0.09 \mathrm{~J} / \mathrm{cm}^{2}$. The fluence of condition (b) is in the correct range to obtain a damage-free layer. 
Figure 2 shows the surface morphology after irradiation of multiple pulses on the same location at $F_{0}=0.07 \mathrm{~J} / \mathrm{cm}^{2}$. This fluence level was chosen as it allowed damage-free processing of the layer when applying single pulses. The time separation between subsequent pulses was on the order of tens of milliseconds or more. This allows the material to cool down before the next shot is applied avoiding inter-pulse heat accumulation. As can be observed from Fig. 2, the molybdenum layer starts to crack when the number of overscans is five. This can be attributed to an increase in absorptivity after the first few pulses, which leads to a high absorbed laser fluence, as described by Eq. (1). Similarly to the single-shot case, if the total absorbed fluence is too high, the layer will be damaged. To account for this effect, the fluence of the individual pulses of a pulse train shall be lower than the single pulse fluence, in order to reach the BDT.

\subsubsection{Single laser tracks}

Based on the results of single and multiple pulses on the same location, lines (i.e. tracks) were produced by applying multiple partly overlapping laser pulses. The effect of spatial pulse-topulse overlap, number of overscans, and laser fluence on the surface morphology of the lines was studied. During these experiments the repetition frequency $f_{p}$ was fixed at either 100 or $200 \mathrm{kHz}$. Figure 4 provides an overview of the experiment matrix. The overlap was calculated for different wavelengths using Eq. (2), where the beam diameter $D$ was used as an input. After initial SEM analysis to identify the conditions which produce LIPSS on damage-free layers, this step was iterated by finer variations of the parameters.

Again, the main goal of this set of experiments was to find conditions which induce the BDT in the layer, as this will result in damage-free textured lines. Whether the BDT was reached was found to mainly depend on the pulse overlap and single pulse fluence as is illustrated in Fig. 5. This figure shows the surface morphologies at constant laser fluence of $0.05 \mathrm{~J} / \mathrm{cm}^{2}$ and as a function of increasing overlap and overscans. The overlap was increased from none (i.e., separate, nonoverlapping pulses) in Fig. 5(a) to a pulse overlap of 97.9\% in Fig. 5(d). As can be observed, the increase of overlap significantly reduces the formation of cracks, delamination and chipping. It was found that only for overlap values over $95 \%$ was the absorbed single pulse fluence $F_{\text {abs }}$, see Eq. (1), in the right range for damage free laser tracks, see Fig. 5(c) and 5(d). This can be explained by the increase in absorption to a steady condition after the first pulses, which shifts condition of Fig. 5(a) to the BDT range shown in Fig. 1.

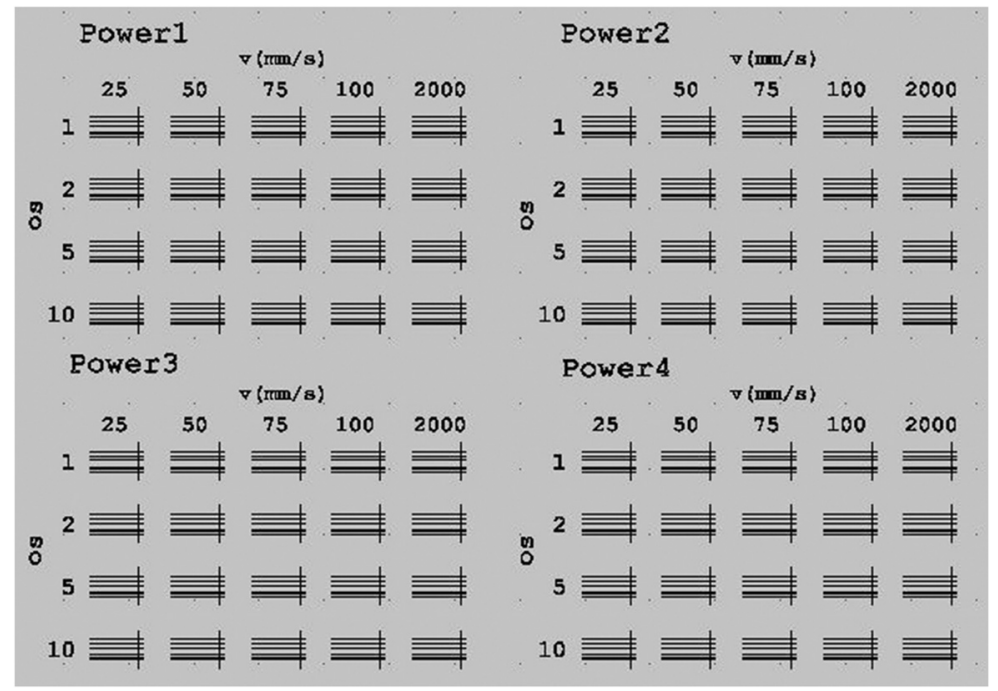

Fig. 4 Experiment matrix used to scan lines with different combinations of irradiation conditions. Repetition frequency was constant, while average laser power/fluence, speed (and by that overlap), and number of overscans (OS) were varied. To check the reproducibility of the results, several identical lines were processed using identical conditions. 


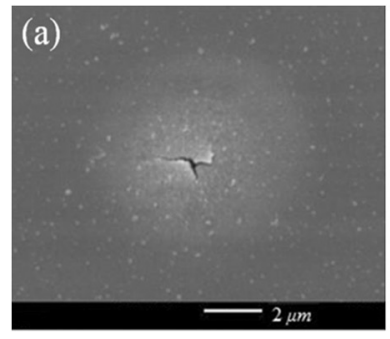

(d)

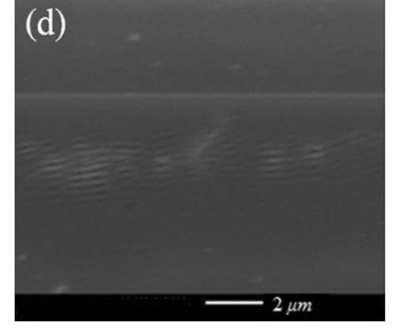

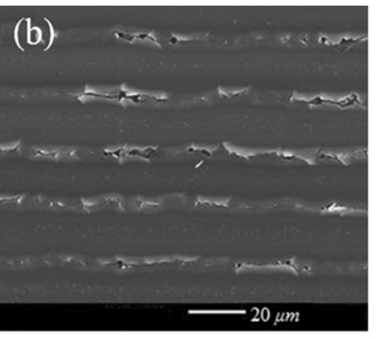

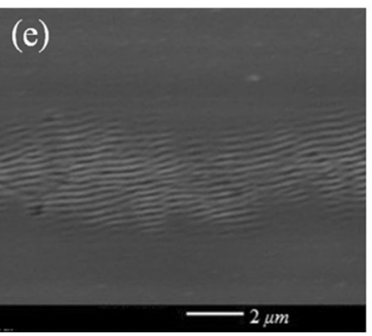

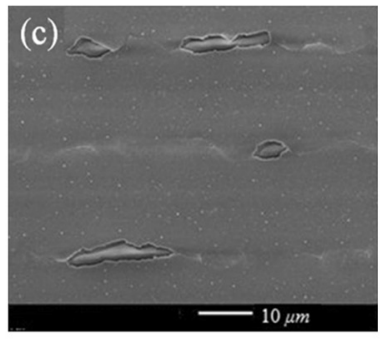

Fig. 5 SEM images of machined $150 \mathrm{~nm}$ PVD deposited molybdenum layers on soda lime glass textured by $6.7 \mathrm{ps}$, UV $(343 \mathrm{~nm})$ laser pulses, where overscans and scanning speed were varied while repetition frequency $(100 \mathrm{kHz})$ and fluence $\left(0.05 \mathrm{~J} / \mathrm{cm}^{2}\right)$ were fixed. It should be noted, that the images are not all to the same scale. (a) Single pulse, (b) 1 overscan, $91.6 \%$ overlap, (c) 1 overscan, $93.7 \%$ overlap, (d) 1 overscan, $97.9 \%$ overlap, (e) 2 overscans, $97.9 \%$ overlap. Only at high pulse overlap is the layer damage-free. The total (or accumulated) fluence deposited in each condition is plotted in Fig. 6 . This set of images clearly shows the effect of the overlap on the fabrication of textured lines without thermal damage. LIPSS starts to grow when $F_{0}^{\text {tot }}$ is increased enough to reach the correct LIPSS fluence range. ${ }^{7}$

When analyzing these results, both the overlap and the overscans (OS) should be accounted for the LIPSS formation, since they only appear in a certain total accumulated fluence domain. ${ }^{7}$ To that end, another quantity is here defined, which is the total optical accumulated laser fluence $F_{0}^{\text {tot }}\left(\mathrm{J} / \mathrm{cm}^{2}\right)$ impinging on the surface of the sample:

$$
F_{0}^{\text {tot }}=F_{0} \times \mathrm{OS} /(1-\mathrm{OL})
$$

which is an approximation valid for $D \gg v / f_{p}$ and OL $>60 \%$ In Eq. (3), $F_{0}$ was used instead of $F_{\text {abs }}\left(F_{0}, N\right)$, which includes the material response to the laser material in the multi-pulse regime; in this sense we are only expressing here the incident fluence on the top surface of the layer.

Figure 6 shows the total fluence $F_{0}^{\text {tot }}$ absorbed at the surface as a function of overlap and overscans, where circles correspond to the processing conditions shown in Fig. 5. Conditions d and $\mathbf{e}$ in Fig. 6 produce damage-free textured tracks on the layer, while conditions indicated by $\mathbf{a}$, $\mathbf{b}$, and $\mathbf{c}$ were found to damage the molybdenum layer.

Similar to what was concluded in Sec. 3.1.1 for single shots, damage-free lines can be attributed to reaching the BDT at each single laser pulse as a result of a reduced surface reflectivity at high overlap. The latter statement is consistent with the observed experimental results shown in Fig. 5(a) and 5(d). Figure 5(a) shows a surface which is damaged by a single laser shot on the untextured surface, but it is not damaged when the overlap is increased in Fig. 5(d). In Fig 5(a) the absorbed fluence $F_{\text {abs }}$ is too low to reach the BDT; however, when the overlap is sufficiently high, the BDT can be reached due to increased absorption at steady processing regime after the first shots of the line. Moreover, the increase of $\mathrm{OL}$ also leads to an increase of $F_{0}^{\text {tot }}$ and entry into the correct range to obtain LIPSS. ${ }^{7}$ In conclusion, the effect of a correct choice of OL, $F_{0}$, and overscans is to keep $F_{\text {abs }}\left(F_{0}, N\right)$ in the correct BDT range for each subsequent shot, while increasing the total deposited fluence $F_{0}^{\text {tot }}$ until the fluence range to obtain LIPSS is reached. This is illustrated in Fig. 1.

To study the effect of pulse duration on the formation of LIPSS, four different pulse durations were applied: 230, 1, 6.7, and 10 ps. For pulse durations of 230 and 1 ps, processing conditions were found which resulted in LIPSS, but the peak-to-valley amplitude of those LIPPS were 


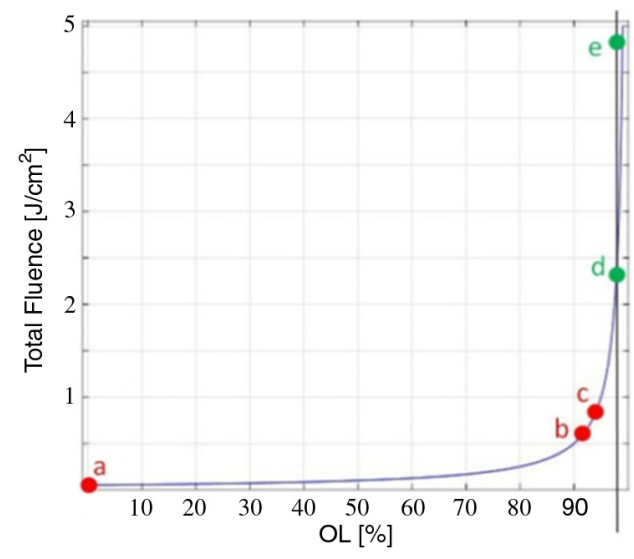

Fig. 6 The total fluence $F_{0}^{\text {tot }}\left(\mathrm{J} / \mathrm{cm}^{2}\right)$ incident on the sample from Eq. (3) is plotted as a function of the overlap (\%) and overscans, at the fixed single pulse average fluence $F_{0}=0.05 \mathrm{~J} / \mathrm{cm}^{2}$. Circles indicate the total fluence of the conditions of the images in Fig. 5. Red circles $\mathbf{a}, \mathbf{b}$, and $\mathbf{c}$ refer to conditions inducing thermal damage of the layer, while green dots $\mathbf{d}$ and $\mathbf{e}$ refer to damage-free conditions.

larger than the thickness of the molybdenum layer. Hence, the glass substrate was exposed in the valleys between peaks in these cases, as seen in Fig. 7. At pulse durations of 6.7 and $10 \mathrm{ps,} \mathrm{the}$ shape of the LIPPS were smoother and more superficial. That is, the valleys were less deep and did not expose the glass carrier. Therefore, to create large areas of LIPSS, the pulse duration in subsequent experiments was fixed to $6.7 \mathrm{ps}$. The fact that these LIPSS were smoother and more superficial can probably be attributed to the relatively short electron-phonon coupling time $\tau_{\text {ep }}$ of molybdenum, when compared to the pulse duration. ${ }^{6,16,17}$ Further study would be required to confirm this.

\subsubsection{Processing areas}

The final step of the experimental procedure consisted of choosing a few sets of processing parameters from the previous step (Sec. 3.1.2), and creating areas of LIPSS by varying the pitch (distance) between parallel lines, which were scanned in the same direction. This pitch is an additional parameter to be considered for texturing areas with LIPSS.

Polarization is an essential factor for determining the direction of regular LIPSS. Depending on the material and machining conditions, this direction can be either parallel or perpendicular to an applied linear polarization. ${ }^{18,19}$ The observed LIPSS on molybdenum thin layers were found to grow perpendicular to the linear polarization of the laser radiation. Long LIPSS, without bifurcations and interruptions, can be obtained if the linear polarization is perpendicular to the

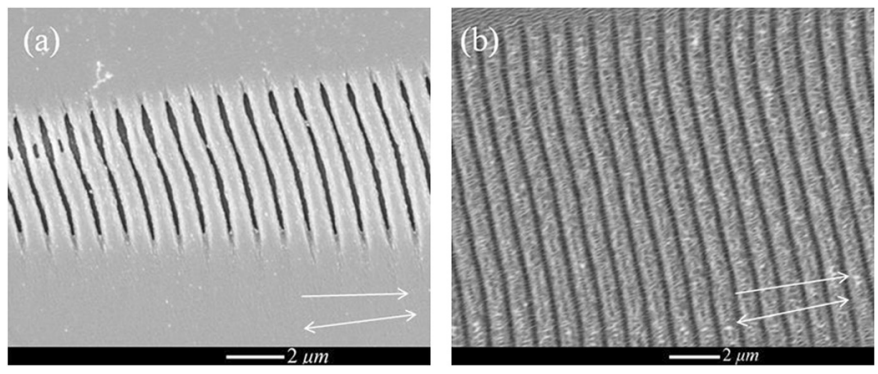

Fig. 7 SEM images of LIPSS in single lines on $400 \mathrm{~nm}$ molybdenum layer irradiated with IR light. (a) $\tau=0.23 \mathrm{ps}$, fluence $=0.81 \mathrm{~J} / \mathrm{cm}^{2}, 93 \%$ overlap, 1 overscan. (b) $\tau=10 \mathrm{ps,} \mathrm{fluence=}$ $1.26 \mathrm{~J} / \mathrm{cm}^{2}, 93 \%$ overlap, 1 overscan. Single arrows in the graphs indicate the processing direction, whereas the double arrows indicate the polarization direction of the laser radiation. LIPSS in (b) are smoother and more superficial than those in (a). 

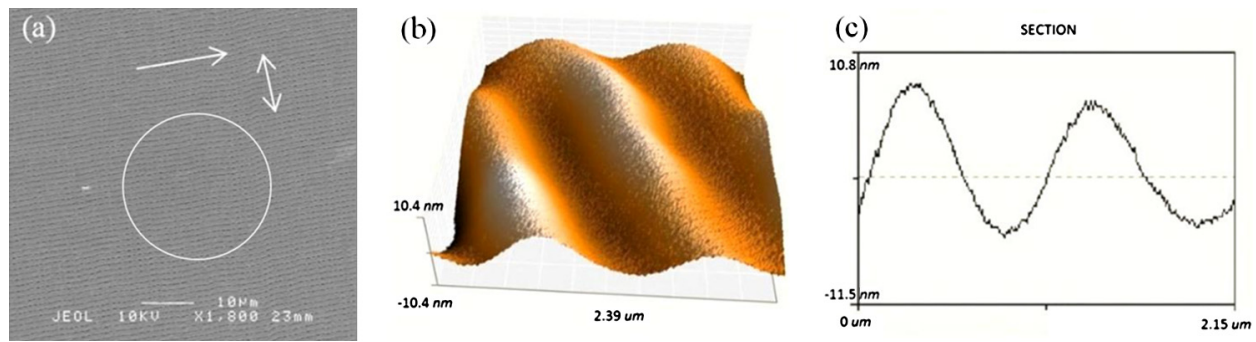

Fig. 8 (a) SEM image of a $870 \mathrm{~nm}$ grating composed by highly periodical LIPSS on $400 \mathrm{~nm}$ molybdenum layer sputtered on soda lime glass. Processing conditions: $\lambda=1030 \mathrm{~nm}, 6.7 \mathrm{ps}, 98 \%$ overlap, $0.013 \mathrm{~J} / \mathrm{cm}^{2}, 100 \mathrm{kHz}, 0.3 \mu \mathrm{m}$ pitch between different lines and $30 \mu \mathrm{m}$ spot size. The LIPSS's length is not limited by the dimensions of the laser spot (indicated by the white circle). Single arrow indicates the scanning direction, while double arrow indicates the polarization. This picture clearly shows that there is no fundamental limitation in the size of the processable area, since the length of the LIPSS is not constrained by the size of the laser's spot. (b) AFM image showing the smooth 3-D topography of the same sample. (c) 2-D cross-section of the surface in Fig. 8(b).

scanning direction. ${ }^{20}$ In this way, LIPSS growth is not constrained by the width of the single laser line and regular nanogratings can be obtained over large areas.

Conditions were found at which the surface was covered with long LIPSS without bifurcations, as shown in Fig. 8(a). It was determined by CLSM that the material removed by ablation on this sample was below $150 \mathrm{~nm}$ in thickness. When required, the thinning of the processed layer could be compensated for by using a thicker layer.

The obtained nanogratings were inspected by SEM and AFM to determine the periodicity and amplitude of the LIPSS obtained with UV, green, and IR laser light (Table 1). It is known from literature that the periodicity of the LIPSS is correlated to the wavelength of the laser. ${ }^{20}$ The results in Table 1 confirm this. It is also known that the amplitude of LIPSS depends on the parameters applied. ${ }^{18,19}$ However, the process window leading to LIPSS on a damage-free thin molybdenum layer is so small that large areas were machined here with just one set of conditions, with respect to each wavelength. For each set of conditions this leads to a single amplitude of the LIPSS, as seen in Table 1. Their 3-D morphology is shown in Fig. 8(b). In addition, Fig. 8(c) shows the smooth sinusoidal shape of its cross-section. Figure 9(a) and 9(b), demonstrates the uniformity of large areas with LIPSS as a uniform color at fixed visual angle due to diffracted light. To confirm the uniformity, Fig. 9(c) shows the AID of the gratings of Fig. 9(a) and 9(b). In Fig. 9(c), two different lobes are visible, one representing the zeroth order and the other representing the first order of diffraction. The first order of diffraction subtracts part of the intensity from the zeroth order as shown in Fig. 9(c). The measured data from Fig. 9(c) matches with a nanograting of $390 \mathrm{~nm}$ periodicity, which is the same value observed with SEM and AFM inspection. As expected for a nanograting of such periodicity, the first order of diffraction disappears at wavelengths longer than the periodicity of the grating. Hence, this confirms that the optical diffraction shown in Fig. 9(a) and 9(b) originates from the LIPSS and not from geometrical features, such as grooves formed by ablation during laser processing. The latter would show a larger periodicity.

Since theoretical and numerical models of LIPSS—or more specifically, models predicting periodicity of LIPSS describing the absorbed laser energy below the rough surface of the material-are known only for bulk material, we compared the periodicity predicted by one of those

Table 1 Averaged periodicity and amplitude (obtained by AFM and SEM) of LIPSS obtained with the three different lasers' wavelengths.

\begin{tabular}{lccc}
\hline \hline & UV $(\lambda=343 \mathrm{~nm})$ & Green $(\lambda=515 \mathrm{~nm})$ & IR $(\lambda=1030 \mathrm{~nm})$ \\
\hline Averaged periodicity $(\mathrm{nm})$ & 270 & 390 & 890 \\
Averaged amplitude $(\mathrm{nm})$ & 29 & 21 & 13 \\
\hline \hline
\end{tabular}



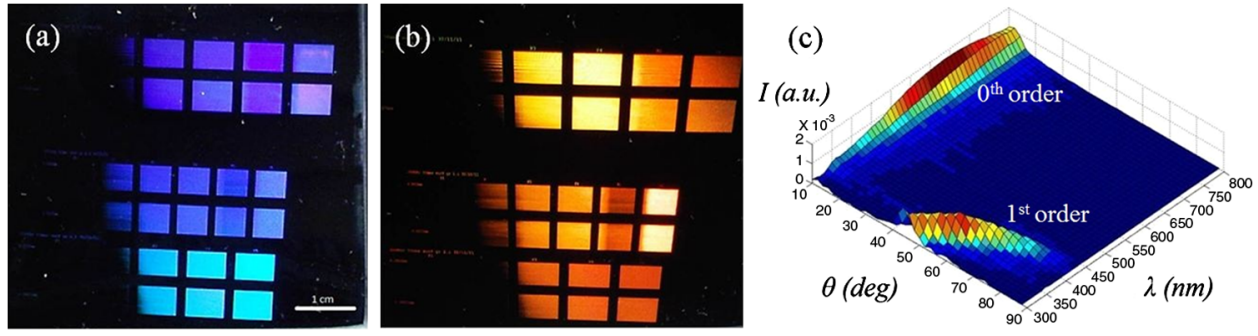

Fig. 9 (a) and (b) Photographs of the same laser textured sample at different angles showing the feasibility of large area nanogratings (LIPSS) on $400 \mathrm{~nm}$ molybdenum layer. (c) AID obtained by spectrophotometry: the intensity $I$ is plotted as function of the scattering angle $\theta$ and the incident light's wavelength $\lambda$. The measurement was taken over an area of $6 \times 6 \mathrm{~mm}^{2}$. Notice that the first order of diffraction subtracts part of the intensity from the zeroth order. Measured data match with a grating of $390 \mathrm{~nm}$ periodicity, confirming that the optical diffracting effect was produced by the LIPSS and not by other geometrical features of larger periodicity.

"bulk" models, known as the efficacy factor theory, ${ }^{21,22}$ to the experimentally obtained periodicity in the thin molybdenum layers. ${ }^{23}$ We found that the periodicity of LIPSS produced on thin films was in good agreement with the theoretical expectations for LIPSS on bulk material.

\section{Conclusions}

By applying ultra-short laser pulses, very regular and damage-free nanogratings consisting of laser-induced periodic surface structures (LIPSS) of various periodicities (890 and $390 \mathrm{~nm}$ ) were fabricated over areas as large as $6 \times 6 \mathrm{~mm}^{2}$ on $400 \mathrm{~nm}$ thin molybdenum films deposited on borosilicate glass. It was found that the optical diffraction effect of the nanograting was indeed produced due to the LIPSS and not by laser ablated grooves of larger periodicity. Furthermore, it was shown that there is no fundamental limitation in the size of the processable area, where the only constraint is given by the beam manipulation system and the size of the sample.

Focusing on the technical side of laser nanofabrication of long regular LIPSS on thin molybdenum layers, the aim of this work was also to give an understanding of the correct laser parameters to apply during laser texturing of the thin layers. It was discussed in detail and demonstrated experimentally that the primary role played by laser parameters, such as fluence and spatial pulse overlap, is to reach the ductile range of molybdenum. Only then thermal damage of the thin layer can be avoided. On the other hand, the laser parameters should be chosen such as to reach the right range of accumulated fluence to initiate LIPSS. It is expected that an analogous approach could be taken to texture thin layers of materials similar to molybdenum, such as chromium and tungsten.

\section{Acknowledgments}

This work was done within the Advanced Dutch Energy Materials Innovation Lab program, funded by the Dutch Ministry of Economic Affairs, Agriculture and Innovation. The authors also want to thank J. Bosman and C. de Kok of Energy Research Center of the Netherlands. Further, the contributions of B. Pathiraj and J. Z. P. Skolski are acknowledged.

\section{References}

1. M. Birnbaum, "Semiconductor surface damage produced by ruby lasers," J. Appl. Phys. 36(11), 3688-3689 (1965), http://dx.doi.org/10.1063/1.1703071.

2. J. F. Young et al., "Laser-induced periodic surface structure. II. experiments on Ge, Si, Al, and brass," Phys. Rev. B 27(2), 1155-1172 (1983), http://dx.doi.org/10.1103/PhysRevB.27 .1155 . 
3. M. Huang et al., "Origin of laser-induced near-subwavelength ripples: interference between surface plasmons and incident laser," ACS Nano 3(12), 4062-4070 (2009), http://dx.doi .org/10.1021/nn900654v.

4. J. Bonse et al., "Ultrashort-pulse laser ablation of indium phosphide in air," Appl. Phys. A 72(1), 89-94 (2001), http://dx.doi.org/10.1007/s003390000596.

5. M. Shinoda et al., "Femtosecond laser-induced formation of nanometer-width grooves on synthetic single-crystal diamond surfaces," J. Appl. Phys. 105(5), 053102 (2009), http://dx .doi.org/10.1063/1.3079512.

6. J. Yao et al., "Selective appearance of several laser-induced periodic surface structure patterns on a metal surface using structural colors produced by femtosecond laser pulses," Appl. Surf. Sci. 258(19), 7625-7632 (2012), http://dx.doi.org/10.1016/j.apsusc.2012.04 .105 .

7. J. Eichstädt et al., "Determination of irradiation parameters for laser-induced periodic surface structures," Appl. Surf. Sci. (2012) (In Press), http://dx.doi.org/10.1016/j.apsusc.2012 .09 .120 .

8. S. K. Lee et al., "Excimer laser ablation removal of thin chromium films from glass substrates," Surf. Coat. Technol. 113(1-2), 63-74 (1999), http://dx.doi.org/10.1016/S0257 -8972(98)00824-X.

9. J. Siegel et al., "UV-laser ablation of ductile and brittle metal films," Appl. Phys. A: Mater. Sci. Process. 64(2), 213-218 (1997), http://dx.doi.org/10.1007/s003390050468.

10. “Molybdenum," http://www.espimetals.com/tech/molybdenum.pdf (August 2012).

11. M. Khantha et al., "Dislocation screening and the brittle-to-ductile transition: a KosterlitzThouless type instability," Phys. Rev. Lett. 73(5), 684-687 (1994), http://dx.doi.org/10 .1103/PhysRevLett.73.684.

12. Y. Jee et al., "Laser-induced damage on single-crystal metal surfaces," J. Opt. Soc. Am. B 5(3), 648-659 (1988), http://dx.doi.org/10.1364/JOSAB.5.000648.

13. W. M. Steen and J. Mazumder, Laser Material Processing, 4th ed., Springer, London (2010).

14. K. Jäger et al., "Angular resolved scattering measurements of nano-textured substrates in a broad wavelength range," Meas. Sci. Technol. 22(10), 105601 (2011), http://dx.doi.org/10 .1088/0957-0233/22/10/105601.

15. J. H. Scofield et al., "Sputtered molybdenum bilayer back contact for copper indium diselenide-based polycrystalline thin-film solar cells," Thin Solid Films 260(1), 26-31 (1995), http://dx.doi.org/10.1016/0040-6090(94)06462-8.

16. Z Lin and L. V. Zhigilei"Electron-phonon coupling and electron heat capacity of metals under conditions of strong electron-phonon nonequilibrium," Phys Rev. B 77(7), 075133 (2008), http://dx.doi.org/10.1103/PhysRevB.77.075133.

17. J. P. Colombier et al., "Effects of electron-phonon coupling and electron diffusion on ripples growth on ultrafast-laser-irradiated metals," J. Appl. Phys. 111(2), 024902 (2012), http://dx .doi.org/10.1063/1.3676221.

18. D. Dufft et al., "Femtosecond laser-induced periodic surface structures revisited: A comparative study on ZnO," J. Appl. Phys. 105(3), 034908 (2009), http://dx.doi.org/10.1063/1 .3074106.

19. E. Rebollar et al., "Ultraviolet and infrared femtosecond laser induced periodic surface structures on thin polymer films," Appl. Phys. Lett. 100(4), 041106 (2012), http://dx.doi .org/10.1063/1.3679103.

20. F. Garrelie et al., "Evidence of surface plasmon resonance in ultrafast laser-induced ripples," Opt. Soc. of Am. 19(10), 9035-9043 (2011), http://dx.doi.org/10.1364/OE.19 .009035 .

21. J. E. Sipe et al., "Laser-induced periodic surface structure. I. theory," Phys. Rev. B 27(2), 1141-1154 (1983), http://dx.doi.org/10.1103/PhysRevB.27.1141.

22. D. C. Emmony et al., "Laser mirror damage in germanium at $10.6 \mu \mathrm{m}, "$ Appl. Phys. Lett. 23(11), 598-601 (1973), http://dx.doi.org/10.1063/1.1654761.

23. D. Scorticati et al., "Thin film surface processing by ultra-short laser pulses," Proc. SPIE 8438, 84380T (2012), http://dx.doi.org/10.1117/12.922270. 


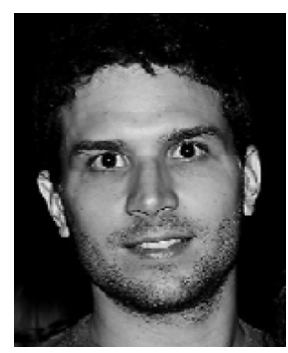

Davide Scorticati received a master's degree in solid state physics at the Università degli Studi di Milano-Bicocca, Italy, in 2010. His internship, focused on the development of new laser scribing processes for thin film photovoltaic solar cells, was carried out at LaserPoint, srl, where he later continued his R\&D activity on new laser processes for industrial applications. He is currently doing his $\mathrm{PhD}$ in the group of Laser Applied Technology at the University of Twente in the Netherlands researching on laser-material interaction for photovoltaics, within the work-frame of the Advanced Dutch Energy Materials Innovation Lab program, funded by the Dutch government.

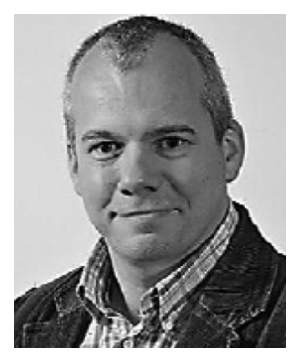

Gert-Willem Römer received his MSc with honors in electrical engineering from the University of Twente in the Netherlands in 1993, and a PhD in mechanical engineering from the same university in 1999. He worked several years in industry as a manager of R\&D developing medical robotics. Currently he is an associate professor at the Chair of Applied Laser Technology at the University of Twente. His scientific focus is on the fundamental study of laser-processes, sensor development, modeling, and real-time control of laser-material processing as applied to laser welding, cladding using high power lasers, as well as micro-machining using ultra short pulsed lasers.

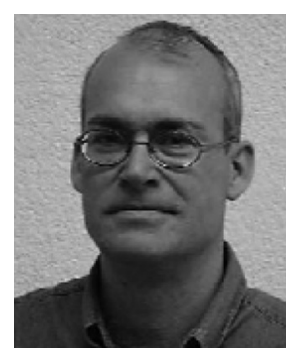

Dirk Frederik de Lange studied mechanical engineering at the University of Twente, finishing his master's degree in the energy processes group, followed by his doctorate in the fluid dynamics group. He worked several years in various projects related to laser-assisted material processing in the laser technology group of the same university and within the Netherlands Institute for Metals Research (NIMR, now known as M2i). Since 2007, he has been a professor at the Universidad Autónoma de San Luis Potosí in Mexico, continuing his research within the field of laser-assisted material processing, as well as a wider range of other manufacturing processes.

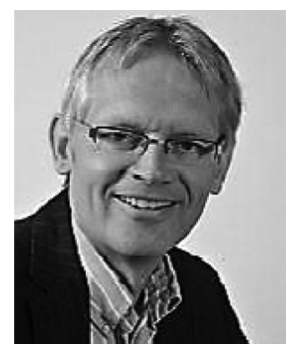

Bert Huis in 't Veld studied physics and obtained a PhD in materials science. He has more than 25 years of experience in surface engineering at TNO Technical Sciences in The Netherlands. He combines a function at TNO in Eindhoven with a position at the University of Twente, where he is leading the applied laser technology group within the Faculty of Engineering Technology. The key issue in his work is the laser-material interaction during micro- and nano-machining using ultra-short laser pulses and during cladding and welding. Both subtractive and additive processes are studied, ranging from scribing and dicing to laser-assisted deposition. 